\title{
Growth morphologies and mechanisms of non-equilibrium solidified MC carbide
}

\author{
Y. Chen ${ }^{\text {a) }}$ \\ Laboratory for Laser Intelligent Manufacturing, Institute of Mechanics, Chinese Academy of \\ Sciences, Beijing 100080, People's Republic of China; and Laboratory of Laser Materials Processing \\ and Surface Engineering, School of Materials Science and Engineering, Beijinghang University, \\ Beijing 100083, People's Republic of China \\ H.M. Wang \\ Laboratory of Laser Materials Processing and Surface Engineering, School of Materials Science and \\ Engineering, Beijinghang University, Beijing 100083, People's Republic of China
}

(Received 13 December 2004; accepted 8 August 2005)

Growth morphologies and mechanisms of the carbide of group IVB and VB elements (MC carbide), a typical faceted crystal, were studied with an estimated cooling rate from $10^{2}$ to $10^{5} \mathrm{~K} / \mathrm{s}$. Results showed that although the growth morphologies of the MC carbide vary remarkably with solidification cooling rate, the solid/liquid interface is always atomically smooth, and the growth mechanisms are always lateral growth. The growth mechanism transition from lateral to continuous growth mode, which was predicted by the classic crystal growth theory, was not observed for the TiC type MC carbide within the estimated cooling rate range of $10^{2}-10^{5} \mathrm{~K} / \mathrm{s}$.

\section{INTRODUCTION}

The nature of the solid/liquid interface structure has a decisive influence on both the growth mechanisms and the growth morphologies of crystals. The Jackson's factor $\alpha_{\mathrm{j}}{ }^{1}$ can be used to predict the solid/liquid interface structure and growth mechanism under slow-cooling, near-equilibrium solidification conditions. For those phases with $\alpha_{j}<2$, the solid/liquid interface structure is widely believed to be atomically rough, and the crystal growth occurs by continuous growth, leading to nonfaceted morphology. For those phases with $\alpha_{j}>2$, the growth interface structure is atomically smooth, and the crystal growth occurs by lateral growth, leading to faceted growth morphologies with special crystallographic planes or facets on the crystal surface. It is predicted and widely accepted that the growth mechanism of a faceted crystal would change from lateral to continuous growth with increasing solidification cooling rate, ${ }^{2}$ as shown in Fig. 1. This prediction is confirmed by some metalloid elements' faceted phases, such as silicon and germanium, ${ }^{4-9}$ with a Jackson's factor slightly over 2 under slow-cooling, near-equilibrium solidification conditions. However, little information is available concerning the

\footnotetext{
a) Address all correspondence to this author. e-mail: chenyao27@163.com

DOI: $10.1557 / J M R .2006 .0043$
}

growth mechanism transition versus solidification conditions for typical faceted crystals with strong atomic bonds and very high Jackson's factor.

As an interstitial phase with high melting point, excellent high-temperature stability and wear resistance, the carbide of group IVB and VB elements (hereafter called MC carbide) such as $\mathrm{TiC}, \mathrm{VC}$, etc, is an important strengthening phase for nickel-based superalloys, alloy tool steels, and many metal matrix composite materials (MMCs). Also, TiC-type MC carbide is well known to be a typical faceted crystal due to high melting entropy and a large Jackson's factor of approximately 5-7. ${ }^{10}$ Therefore, investigation on the non-equilibrium solidification growth morphologies of MC carbide is very important to perfect understanding of the non-equilibrium growth theory of faceted crystals. The objective of this work was to investigate the growth morphologies of $\mathrm{TiC}$ with an estimated cooling rate from $2.1 \times 10^{2}$ to $8.3 \times 10^{5} \mathrm{~K} / \mathrm{s}$.

\section{EXPERIMENTAL PROCEDURES}

To eliminate the influence of the melt inheritance on the carbide growth morphology, laser surface in situ alloying with carbon was utilized to synthesize TiC-type $\mathrm{MC}$ carbide on the surface of a $\gamma$-titanium aluminide alloy $\mathrm{Ti}-48 \mathrm{Al}-2 \mathrm{Cr}-2 \mathrm{Nb}$. Commercially pure carbon powders, of approximately 320 mesh particle size and approximately $0.2 \mathrm{~mm}$ in thickness, were pre-placed on the surface of the $\gamma$-TiAl intermetallic alloy 


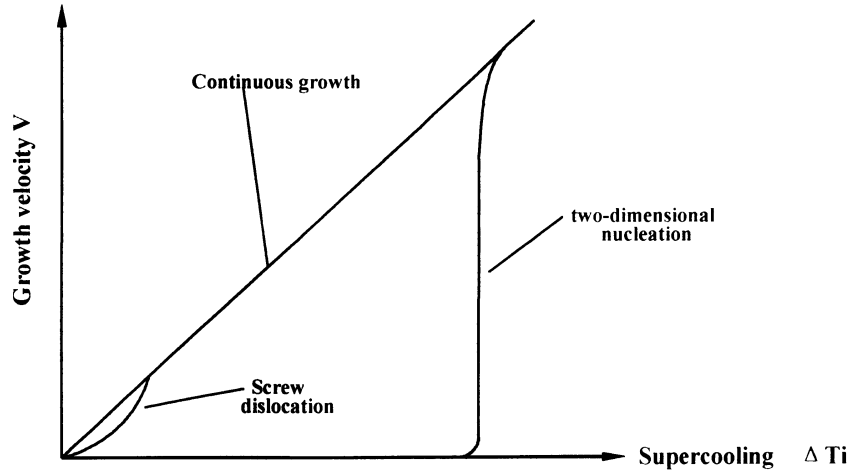

FIG. 1. Growth rate versus interface supercooling according to the three classical laws of crystal. ${ }^{3}$

$(12 \times 10 \times 6 \mathrm{~mm}$ in size $)$. The experiments of laser surface alloying were carried out on a $5 \mathrm{~kW} \mathrm{CO}_{2}$ laser materials processing system equipped with a 4 -axis computer controlled (CNC) work table. The laser processing parameters were laser beam power, $3.2 \mathrm{~kW}$; beam diameter, $3.0 \mathrm{~mm}$; and beam scanning speed, $6.0-16.4 \mathrm{~mm} / \mathrm{s}$.

To obtain higher solidification cooling rate, the growth morphologies of TiC-type MC carbide of pulsed laser surface alloyed $\gamma$-TiAl with carbon were also investigated. The pure carbon powders with an average particle size of 320 mesh were pre-placed on the surface of the $\gamma$-TiAl intermetallic alloy $(12 \times 10 \times 6 \mathrm{~mm}$ in size $)$ in thickness of approximately $0.1 \mathrm{~mm}$. The $1 \mathrm{~kW} \mathrm{Nd:YAG}$ laser was used, and the single-spot laser surface alloying parameters were laser outpower, $17 \mathrm{~J}$; beam diameter, $1.0 \mathrm{~mm}$; and pulse time, $2.0-4.0 \mathrm{~ms}$.

Metallographic sections of the laser surface alloyed coatings were prepared by standard mechanical polishing procedures. The growth morphologies of TiC-type MC carbide were characterized by scanning electron microscopy (SEM; KYKY-2800, KYKY Technology Development Ltd., Beijing, People's Republic of China). The average solidification cooling rate of the laser surface alloyed coating were estimated based on the empirical relationship between secondary dendrite arm spacing and solidification cooling rate. ${ }^{11}$

\section{RESULTS}

As shown in Fig. 2(a), the growth morphology of TiC carbide with a solidification cooling rate of approximately $2.1 \times 10^{2} \mathrm{~K} / \mathrm{s}$ is a well-developed dendrites having platelet/catenulate growth characteristics. It can be clearly seen that the dendrite arms consist of many platelets, which form the basis growth units, parallel to each other. Meanwhile, some thin rods with strong faceted fronts are found to grow out from the external planes of three growth units with a branching angle of approximately $72^{\circ}$ or $108^{\circ}$. The growth morphologies of TiC-type MC carbide in the laser surface alloyed coating on $\gamma$-TiAl intermetallic alloy with a solidification cooling rate of approximately $8.9 \times 10^{2} \mathrm{~K} / \mathrm{s}$ is also

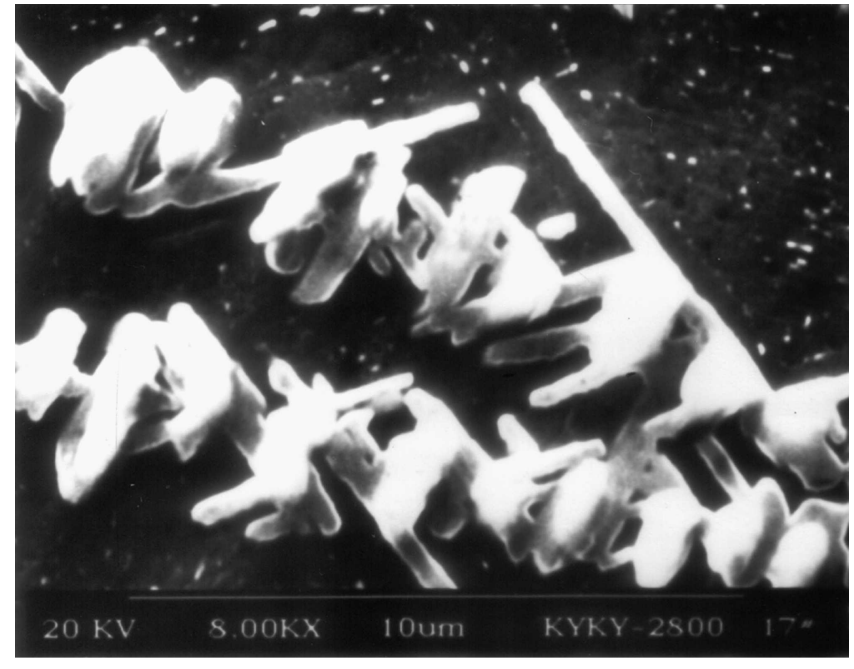

(a)

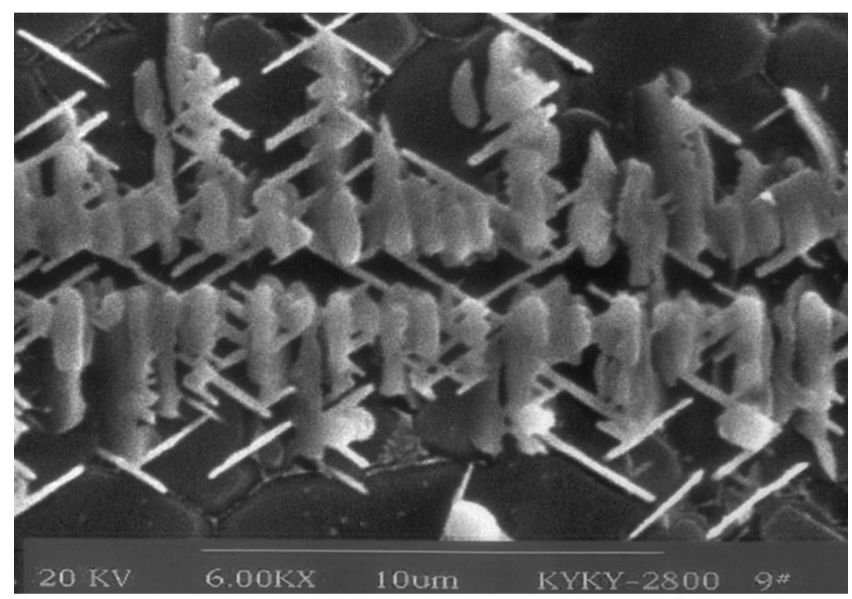

(b)

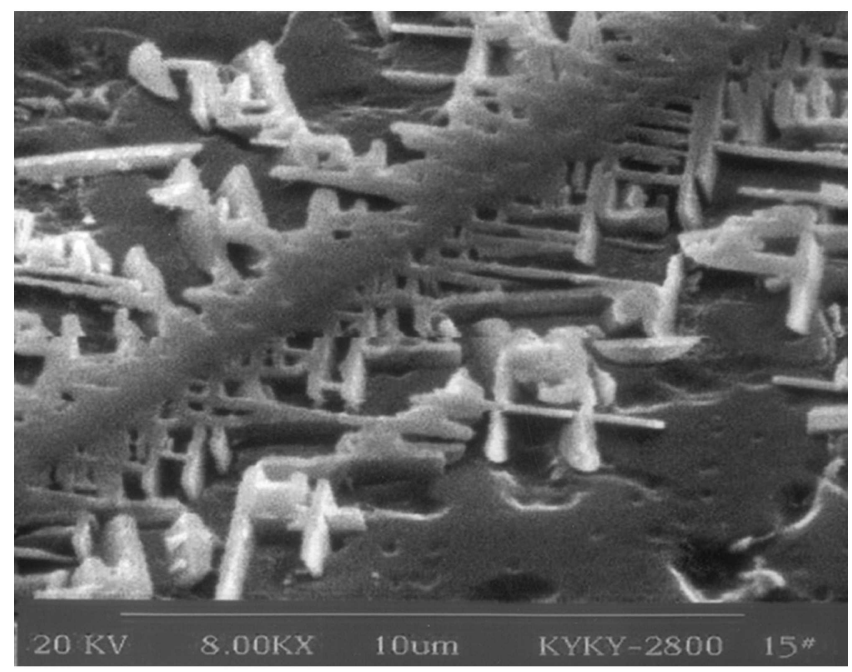

(c)

FIG. 2. SEM micrographs showing the growth morphologies of $\mathrm{TiC}$ of $\mathrm{CO}_{2}$ laser surface alloyed $\gamma$-TiAl intermetallic alloy with carbon solidified with estimated cooling rate of (a) $2.1 \times 10^{2} \mathrm{~K} / \mathrm{s}$ and (b), (c), $8.9 \times 10^{2} \mathrm{~K} / \mathrm{s}$. 


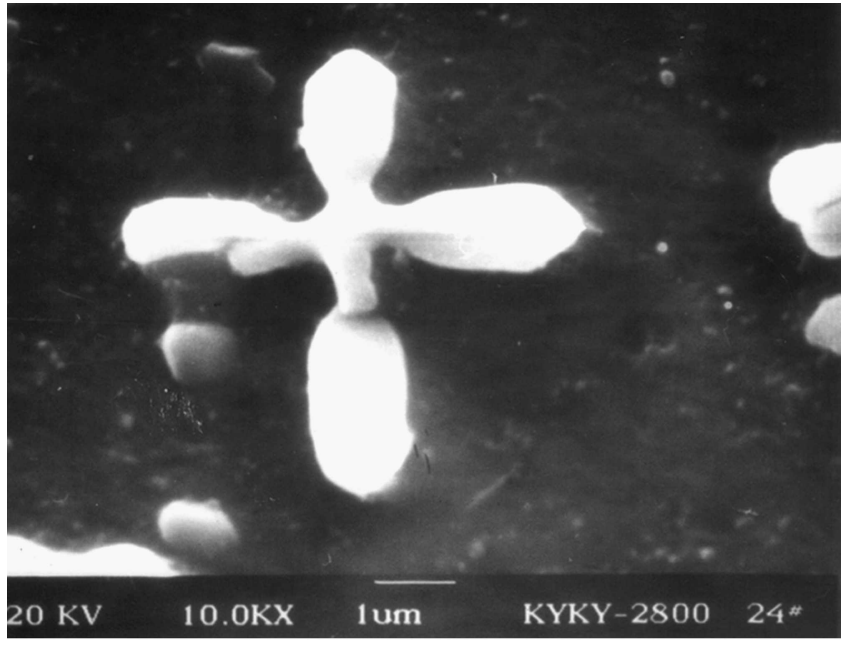

(a)

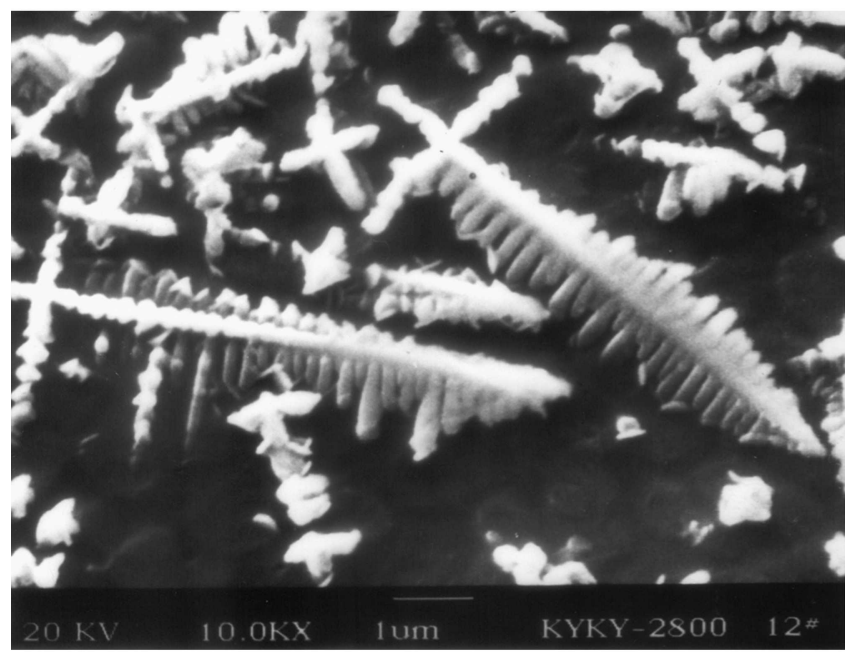

(b)

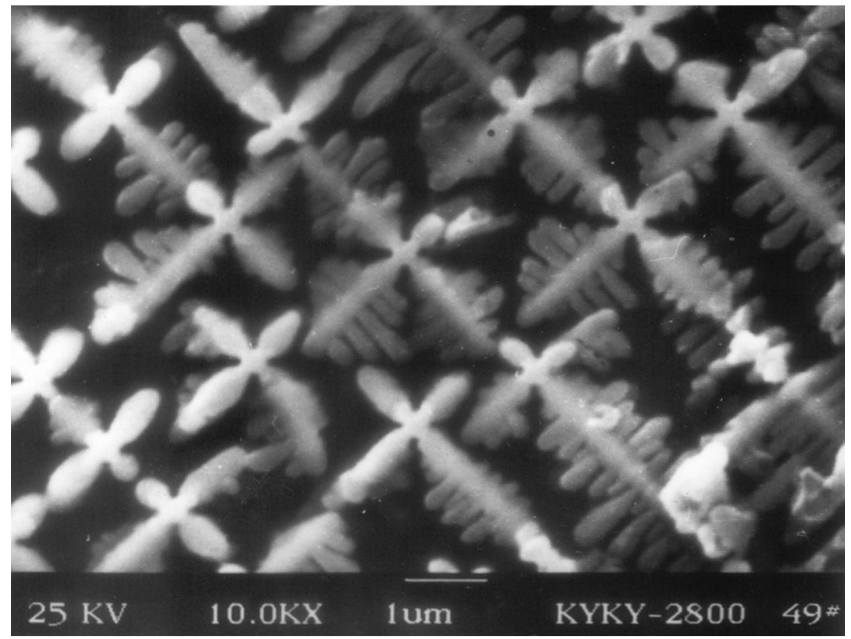

(c)

FIG. 3. SEM micrographs showing the growth morphologies of TiC laser surface of Nd:YAG alloyed $\gamma$-TiAl intermetallic alloy with carbon solidified with estimated cooling rate of (a) $3.7 \times 10^{3} \mathrm{~K} / \mathrm{s}$, (b) $7.1 \times 10^{5} \mathrm{~K} / \mathrm{s}$, and (c) $8.3 \times$ $10^{5} \mathrm{~K} / \mathrm{S}$. macroscopically well-developed dendrite [Fig. 2(b)]. Careful examination of the well-developed dendrite reveals that the primary dendrite arm actually consists of interconnecting faceted rods or faceted rod frameworks, as shown in Fig. 2(b). Moreover, the intersecting angles between these interconnecting thin rods are also approximately $72^{\circ}$ or $108^{\circ}$, equal to the intersecting angles between the close packed crystallographic planes of $\{111\}$ of TiC carbide. Thus, the growth surfaces of thin faceted rods are probably the close-packed $\{111\}$ planes, and these thin rods grow and bifurcate by lateral growth from steps or ledges existing on the surface of these close packed crystallographic $\{111\}$ planes. Further careful observation of the carbide growth morphology on deeply etched specimen indicates that all the outer surfaces of these thin rods have typical facets, as seen clearly in Fig. 2(c).

As solidification cooling rate increases to $3.7 \times$ $10^{3} \mathrm{~K} / \mathrm{s}$, the growth morphology of MC carbide changes to cross-petal-like, and these secondary arms grow symmetrically, as shown in Fig. 3(a). Also, it is worth noting that the growth surfaces still have typically faceted features, as indicated in Fig. 3(a). The growth morphology of TiC solidified with a cooling rate of $7.1 \times$ $10^{5} \mathrm{~K} / \mathrm{s}$ is a dendrite, in which a primary arm grows rapidly along its preferred growth crystallographic direction [Fig. 3(b)]. It is interesting that the advancing front of the secondary arms is still faceted feature, as shown in Fig. 3(b). As the solidification cooling rate increases further to $8.3 \times 10^{5} \mathrm{~K} / \mathrm{s}$, the carbide growth morphology is cross-petal-like [Fig. 3(c)], which is similar to that solidified with a cooling rate of $3.7 \times 10^{3} \mathrm{~K} / \mathrm{s}$. Compared with the carbide growth morphology solidified with a cooling rate of $3.7 \times 10^{3} \mathrm{~K} / \mathrm{s}$, the carbide size with an estimated cooling rate of $8.3 \times 10^{5} \mathrm{~K} / \mathrm{s}$ is clearly decreased, and secondary arms present anisotropy of growth velocity. However, then the faceted characteristics on the growing front of arms can be seen in Fig. 3(c), strongly suggesting that the growth of TiC even under rapid solidification conditions with an estimated cooling rate up to $8.3 \times 10^{5} \mathrm{~K} / \mathrm{s}$ is still by the lateral spreading mechanism and does not transform to the continuous growth mechanism.

In summary, although the growth morphologies of TiC-type MC carbide vary considerably with increasing solidification cooling rate estimated from $10^{2}$ to $10^{5} \mathrm{~K} / \mathrm{s}$, the growth surfaces of carbide all have typical faceted features. This illustrates that TiC-type MC carbide, a typical faceted crystal, grows by the mechanism of faceted growth from the ledges or steps over a very broad range of solidification cooling rate.

\section{DISCUSSION}

It is well understood that morphological change is primarily attributed to the change in growth mechanisms, 


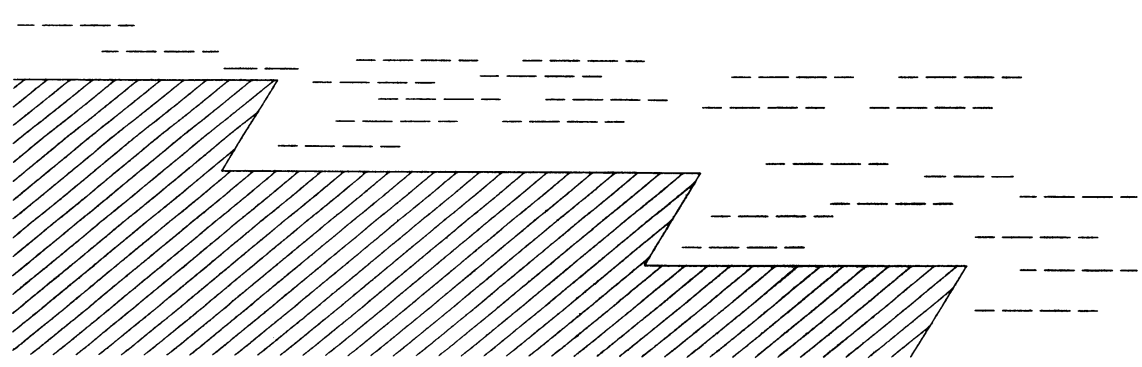

(a)
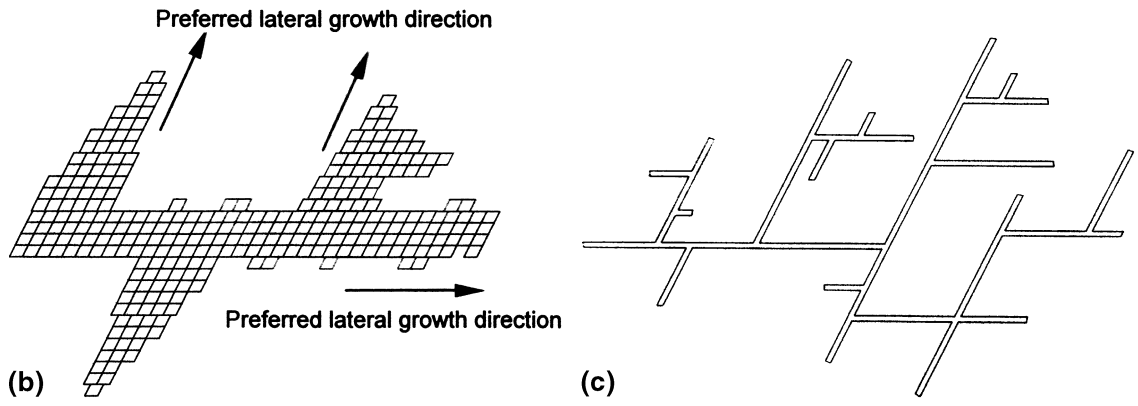

FIG. 4. Schematic illustration of the effect of solidification cooling rate on solid/liquid interface structure for a typical faceted crystal: (a) the solid/liquid interface with large growth steps under slow-cooling solidification conditions, (b) solid/liquid interface with high density of fine growth steps under solidification conditions with a higher solidification cooling rate, and (c) branching of a faceted TiC by alternating lateral growth along the two preferred lateral growth directions.

which are directly correlated to the solid/liquid interface structures, either atomically smooth or rough. ${ }^{9}$ TiC-type $\mathrm{MC}$ carbide is well known to have the NaCl-type crystal structure, and its overall preferred growth direction is $\langle 001\rangle$ by the micro-mechanisms of lateral growth from the steps on the growing surface. Under equilibrium and/ or conventional solidification conditions, the atomic diffusion of $\mathrm{TiC}$ forming elements in the melt is sufficient so that higher index planes grow most rapidly, leaving the close-packed crystallographic planes as the facets on the crystal surface, as shown in Fig. 4(a). As the solidification cooling rate or undercooling increases, the carbide-forming element diffusion required to form the equilibrium carbide growth morphology becomes more difficult. The density of growth steps on the solid/liquid interface also increases with cooling rate or undercooling due to a dramatic increase in propagation of crystal defects such as screw dislocations and twins on the growing interface, ${ }^{12}$ resulting in more steps on the solid/liquid interface, as shown in Fig. 4(b). Because the side walls of these growth steps are always low-energy close-packed crystallographic planes to ensure the lowest Gibbs free energy, atoms from the melt still cannot deposit directly on the atomically smooth solid/liquid interface, and the re-entrant corners produced by side walls of growth steps are the most preferential sites for atomic attachment from the lowest interfacial energy point of view. It is necessary to note that growth of $\mathrm{TiC}$ along the preferred direction would cease because of either the effect of the diffusion of solute and heat in front of solid/liquid interface or the restriction of the growth space. The final growth morphologies of $\mathrm{TiC}$ are either regular branching faceted dendrites or cross-petal-like [Fig. 4(c)].

\section{CONCLUSIONS}

The growth morphologies of TiC-type MC carbide were studied under rapid solidification conditions as a function of estimated cooling rate from $2.1 \times 10^{2}$ to $8.3 \times 10^{5} \mathrm{~K} / \mathrm{s}$. Although growth morphologies of TiC precipitated from different compositional liquid vary remarkably with solidification cooling rate in the estimated range of $10^{2}-10^{5} \mathrm{~K} / \mathrm{s}$, the advancing fronts are always faceted. This illustrates that $\mathrm{TiC}$ grows by lateral growth mechanism even though $\mathrm{TiC}$ solidifies at an estimated cooling rate of $8.3 \times 10^{5} \mathrm{~K} / \mathrm{s}$.

\section{ACKNOWLEDGMENTS}

The authors would like to acknowledge the financial support of the National Natural Science Foundation of China (Grant No. 59971003) and the Trans-Century Training Program for outstanding Talents of the Ministry of Education of China.

\section{REFERENCES}

1. K.A. Jackson: Crystal growth kinetics. Mater. Sci. Eng. 65, 7 (1984). 
2. J.W. Cahn, W.B. Hillings, and G.W. Sears: The molecular mechanism of solidification. Acta Metall. 12, 1421 (1964).

3. H. Biloni: Solidification, in Physical Metallurgy, Part I, edited by R.W. Cahn and P. Haasen (North-Holland Physics Publishing, Amsterdam, The Netherlands, 1983), p. 491.

4. D. Li and D.M. Herlach: Direct measurements of free crystal growth in deeply undercooled melts of semiconducting materials. Phvs. Rev. Lett. 77, 1801 (1996).

5. P.V. Evans, S. Vitta, R.G. Hamerton, A.L. Geer, and D. Turnbull: Solidification of germanium at high undercoolings: Morphological stability and the development of grain structure. Acta Mater. 38. 233 (1990).

6. R-Y. Wang, W-H. Lu, and L.M. Hogan: Growth morphology of primary silicon in cast $\mathrm{Al}-\mathrm{Si}$ alloys and the mechanism of concentric growth. J. Crvst. Growth 207, 43 (1999).
7. L.V. Mikheev and A.A. Chernov: Mobility of a diffuse simple crystal-melt interface. J. Crvst. Growth 112, 591 (1991).

8. R-P. Liu, W-K. Wang, D. Li, and D.M. Herlach: Transition from continuous to lateral growth for Ge crystal in undercooled $\mathrm{Ge}_{74} \mathrm{Ni}_{26}$ alloy melts. Scripta Mater. 41, 855 (1999).

9. R-P. Liu, T. Volkmann, and D.M. Herlach: Undercooling and solidification of Si by electromagnetic levitation. Acta Mater. 49. 439 (2001)

10. H-Z. Fu, L. Liu, and X-H. Fang: Study on the growth law of MC carbide in Ni-base superalloy. J. North. Polytech. Univ. 5, 279 (1987, in Chinese).

11. M. Bass: Laser Materials Processing (North-Holland, Amsterdam, The Netherlands, 1983), pp. 235-295.

12. K.A. Jackson: Liquid Metals and Solidification (ASM, Cleveland, $\mathrm{OH}, 1958)$, p. 181. 